\title{
Impacto da variabilidade de tarefas nas distribuições de aplicações baseadas em tarefas
}

\author{
Lucas Leandro Nesi ${ }^{1 *}$, Arnaud Legrand ${ }^{2}$, Lucas Mello Schnorr $^{1}$ \\ ${ }^{1}$ Instituto de Informática - Universidade Federal do Rio Grande do Sul (UFRGS) \\ Caixa Postal 15.064 - 91.501-970, Porto Alegre - RS - Brasil \\ ${ }^{2}$ Univ. Grenoble Alpes, CNRS, Inria, Grenoble INP, LIG \\ F-38000, Grenoble - France \\ \{lucas.nesi, schnorr\}@inf.ufrgs.br, arnaud.legrandeimag.fr
}

\begin{abstract}
Resumo. Algoritmos de distribuição de dados e tarefas em vários nós computacionais podem utilizar o poder de processamento de cada nó como parâmetro. O poder de cada nó pode ser calculado utilizando uma tarefa predominante. Entretanto, estas tarefas estão sujeitas a variabilidade. Este trabalho investiga o desempenho das distribuições estáticas quando as tarefas sofrem variabilidade.
\end{abstract}

\section{Introdução}

Aplicações paralelas distribuídas requerem um mapeamento de dados e processamento entre as máquinas, de forma a distribuir a carga de trabalho e minimizar o tempo total de execução. A programação de distribuições não cíclicas [Nesi et al. 2020] é mais trabalhosa em paradigmas tradicionais como MPI. Entretanto, a programação baseada em tarefas apresenta uma flexibilidade, já que pode usar um runtime para controlar a memória de uma distribuição informada. Aplicações baseadas em tarefas são organizadas em um DAG (Grafo Acíclico Dirigido). Um runtime, como StarPU [Augonnet et al. 2011], recebe a submissão de tarefas e escalona dinamicamente para os recursos. No caso do StarPU, a distribuição dos dados em diferentes nós computacionais é estática e informada pelo programador, para melhorar a escalabilidade. Exemplos de distribuição para a álgebra linear incluindo a fatoração LU, usada neste trabalho, é o algoritmo 1D-1D para recursos heterogêneos [Beaumont et al. 2001] e extensões [Nesi et al. 2020]. A distribuição 1D-1D usa o poder de processamentos das máquinas que pode ser calculada utilizando o tempo da tarefa predominante na operação. No LU é a tarefa de multiplicação de matrizes (dgemm). Entretanto, o tempo de execução das tarefas pode sofrer variabilidade.

A variabilidade das tarefas pode ter várias causas: A estocasticidade da plataforma, acessos a memória e estados diferentes de cache podem causar uma variabilidade de curto prazo, onde durante uma operação de álgebra linear, a fatoração LU por exemplo, os kernels podem sofrer variação. Entretanto, o escalonamento dinâmico intra nó do StarPU cuida deste problema. Uma outra situação é a variabilidade de longo prazo, quando os kernels apresentam variabilidade entre as operações de álgebra linear ou em dias (tempo) de execução distintos. Isto pode acontecer por aquecimento do hardware e sua má instalação [Cornebize and Legrand 2019]. Este trabalho visa investigar se uma distribuição dinâmica é necessária por causa da variabilidade de longo prazo. Ou se distribuições estáticas que são computadas com o tempo médio das tarefas são suficientes.

*Bolsista do Conselho Nacional de Desenvolvimento Científico e Tecnológico (CNPq). 


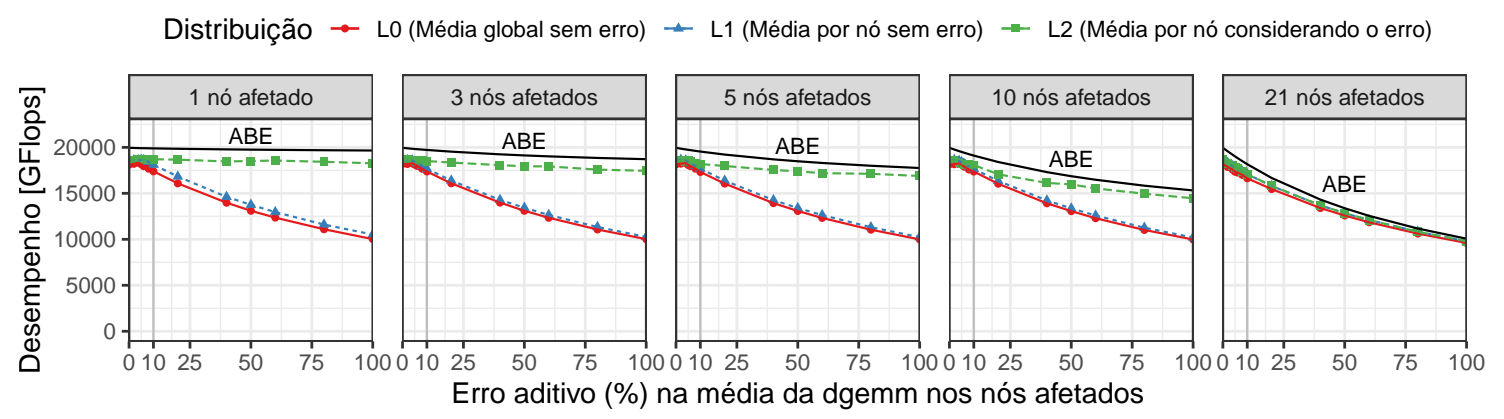

Figura 1. Desempenho das distribuições em função do erro aditivo na tarefa

Para analisar a interferência desta variabilidade foi utilizada a aplicação Chameleon [Agullo et al. 2010] e a operação LU com o StarPU-Simgrid e modelos de desempenho de 21 nós reais. Considerado cinco cenários onde uma certa quantidade de nós $(1,3,5,10,21)$ apresenta um erro aditivo (0\%-100\%) na média de tempo de execução da dgemm, criando uma variabilidade sintética. Por exemplo, no cenário com um nó afetado, com um erro de $50 \%$, se a média da execução da dgemm duraria $50 \mathrm{~ms}$, ela vai durar $75 \mathrm{~ms}$. Para cada cenário e erro aditivo, três distribuições são calculadas: L0 - onde é utilizada a média da dgemms sem erro aditivo de todas as máquinas para calcular a distribuição (representando uma calibragem antiga de apenas uma máquina para gerar a distribuição), L1 - onde é utilizada a média das dgemms sem erro aditivo por máquina para gerar a distribuição (representando uma calibragem antiga por máquina). L2 - onde é utilizada a média da dgemms com o erro aditivo (representado uma calibragem pré operação da distribuição). A Figura 1 apresenta os resultados de desempenho (GFlops) em função do erro aditivo para cada distribuição, com um upper bound de desempenho (ABE).

O principal aspecto dos resultados é a distância de L1 para L2. Se a perda de desempenho entre L1 e L2 for muito grande, é necessário a utilização de outras estratégias, como balanceamento dinâmico da distribuição por exemplo. Assim uma distribuição L1 seria dinamicamente balanceada para ter desempenhos semelhantes às distribuições L2. Entretanto, a diferença só acontece de maneira significativa para erros superiores a $10 \%$ e a maior variabilidade encontrada de longo prazo na literatura é inferior a $10 \%$ [Cornebize and Legrand 2019]. Desta maneira não fica evidenciado a necessidade de balanceadores dinâmicos entre os nós nesta aplicação. A utilização de calibragens antigas ou anteriores a execução da aplicação é suficiente para gerar uma distribuição estática.

\section{Referências}

Agullo, E. et al. (2010). Faster, Cheaper, Better - a Hybridization Methodology to Develop Linear Algebra Software for GPUs. In GPU Computing Gems. Morgan Kaufmann.

Augonnet, C. et al. (2011). StarPU: A Unified Platform for Task Scheduling on Heterogeneous Multicore Architectures. Conc. Comp.: Pract. Exp., SI:EuroPar 2009, 23.

Beaumont, O., Legrand, A., Rastello, F., and Robert, Y. (2001). Static LU decomposition on heterogeneous platforms. Int. Journal of High Performance Comp. Applications.

Cornebize, T. and Legrand, A. (2019). DGEMM performance is data-dependent. Research Report RR-9310, Université Grenoble Alpes ; Inria ; CNRS.

Nesi, L. L., Schnorr, L. M., and Legrand, A. (2020). Communication-Aware Load Balancing of the LU Factorization over Heterogeneous Clusters. In 2020 IEEE 26th ICPADS. 